\title{
Comparação do efeito do metronidazol tópico $10 \%$ associado à nifedipina tópica $0,3 \%$ versus nifedipina tópica $0,3 \%$ isolada no pós-operatório de hemorroidectomia pela técnica híbrida
}

\author{
Comparison of effect of topical metronidazole $10 \%$ associated with nifedipine \\ $0.3 \%$ versus nifedipine $0.3 \%$ alone in postoperative \\ hemorrhoidectomy by hybrid technique
}

\author{
MARCELO PANDOLFI BASSO ${ }^{1}$, ADRIANA BORGONOVI CHRISTIANO ${ }^{1}$, MARCELO RODOLFO MARCIANO $^{1}$, \\ GENI SATOMI CUNRATH², JOÃO GOMES NETINHO ${ }^{3}$
}

${ }^{1}$ Médico Coloproctologista da Faculdade de Medicina de São José do Rio Preto (FAMERP) - São José do Rio Preto (SP), Brasil.
${ }^{2}$ Médica Assistente da Disciplina de Coloproctologia do Hospital de Base da FAMERP - São José do Rio Preto (SP), Brasil.
${ }^{3}$ Chefe da Disciplina de Coloproctologia do Hospital de Base da FAMERP - São José do Rio Preto (SP), Brasil.

BASSO MP, CHRISTIANO AB, MARCIANO MR ,CUNRATH GS, NETINHO JG. Comparação do efeito do metronidazol tópico $10 \%$ associado à nifedipina tópica $0,3 \%$ versus nifedipina tópica $0,3 \%$ isolada no pós-operatório de hemorroidectomia pela técnica híbrida. Rev bras Coloproct, 2011;31(2): 131-138.

RESUMO: A hemorroidectomia é uma operação frequente e considerada dolorosa para a maior parte dos pacientes. Metronidazol e nifedipina tópicos têm demonstrado diminuir a dor no pós-operatório, quando aplicados isoladamente. $O$ objetivo deste estudo foi avaliar o efeito do metronidazol e nifedipina tópicos, em conjunto, na redução da dor pós-operatória, na evacuação, no desenvolvimento de plicomas inflamatórios e complicações. Material e métodos: Foi feito um estudo duplo-cego, prospectivo e randomizado, incluindo 42 pacientes. $O$ Grupo 1 utilizou metronidazol $10 \%$ associado à nifedipina $0,3 \%$ e o Grupo 2 apenas nifedipina $0,3 \%$. A dor foi avaliada utilizando uma escala visual analógica (VAS) nas 12 horas do pós-operatório e nos dias 1, 4, 14 e 28. Resultados: Os pacientes que utilizaram a combinação de medicamentos tópicos tiveram menos dor, menor necessidade de analgesia e dose ingerida de analgésicos, maior precocidade evacuatória, menor desenvolvimento de plicomas inflamatórios e complicações, do que aqueles que usavam nifedipina tópica isoladamente; porém, as diferenças estatísticas não foram significativas. Conclusão: A adição de metronidazol à nifedipina na forma tópica não melhorou os parâmetros avaliados no estudo; no entanto, ambos os tratamentos são justificados para uso no pós-operatório de hemorroidectomia.

Palavras-chave: dor pós-operatória; metronidazol; hemorroidas; hemorroidectomia.

\section{INTRODUÇÃO}

Hemorroidas são plexos vasculares internos e externos, cobertos por mucosa, localizado no espaço submucoso do canal anal, acima ou abaixo da linha denteada, respectivamente, e são parte da anatomia normal do ser humano ${ }^{1,2}$. O tratamento cirúrgico des- tas vem diminuindo com o passar dos anos em virtude do avanço no tratamento não-operatório, que consiste em medidas clínicas e procedimentos ambulatoriais menores, como a ligadura elástica.

Quando indicada, a hemorroidectomia pode ser realizada pela técnica aberta, fechada, semifechada ou híbrida, em que o tecido hemorroidário é ressecado, sendo

Trabalho realizado no Hospital de base da Faculdade de Medicina de São José do Rio Preto (FAMERP), São José do Rio Preto (SP), Brasil.

Fonte de financiamento: não há

Conflito de interesse: nada a declarar 
que, nesta última, o componente interno é ligado e o externo é suturado conforme técnica fechada (Fergunson). Há ainda um procedimento com a utilização do grampeador circular, melhor denominado hemorroidopexia, quando o tecido hemorroidário não é retirado, mas sim reposicionado na sua situação anatômica original ${ }^{2,3}$.

Independentemente da técnica, a dor é a principal complicação pós-operatória da cirurgia. O gerenciamento desta é o maior desafio para os cirurgiões colorretais $^{4-10}$.

A gênese da dor no pós-operatório de hemorroidectomia não apresenta causa bem definida. Esta parece ser multifatorial, com dependência da tolerância individual, modo de anestesia, analgesia pós-operatória e técnica cirúrgica ${ }^{11}$.

Porém, a resposta inflamatória local, secundária ao espasmo esfincteriano, incisão cirúrgica e infiltração bacteriana da ferida cirúrgica seriam fatores principais nesse contexto ${ }^{8-11}$. Dentre as medicações, que proporcionam bloqueio destas vias, estão o metronidazol e a nifedipina.

Apesar de seu efeito contra protozoários e bactérias anaeróbicas, o metronidazol apresenta ação antiinflamatória. Logo, a diminuição da dor ocorre devida suas propriedades antibióticas, com diminuição da colonização bacteriana e por seu efeito contra flogose ${ }^{8}$.

Já, a nifedipina consiste em um inibidor do canal de cálcio, que diminui o influxo de cálcio para a musculatura lisa esfincteriana perianal, com alteração do mecanismo de contração das fibras musculares e diminuição da pressão local. O relaxamento da musculatura melhora o aporte sanguíneo e a cicatrização local. Ou seja, o decréscimo da dor se obtém por diminuição do espasmo esfincteriano e pela cicatrização da ferida ${ }^{1,10}$.

Nenhum estudo avaliou o benefício da associação de ambas as medicações no período pós-operatório de hemorroidectomia. $\mathrm{O}$ objetivo deste trabalho consiste na avaliação da dor, necessidade de uso de analgésicos, evacuação, presença de plicoma inflamatório e complicações de pacientes submetidos à hemorroidectomia pela técnica híbrida, que usaram nifedipina tópica $0,3 \%$ associada ao metronidazol $10 \%$, em relação a nifedipina $0,3 \%$ tópica isolada (ambos sob a forma de apresentação de pomada), no periodo pós-cirúrgico de 28 dias.

Não utilizamos um terceiro grupo comparativo sem o uso de ambas as medicações, visto que a literatura médica demonstra o benefício da nifedi- pina no pós-operatório de hemorroidectomia ${ }^{1,10}$. Assim, tentamos demonstrar um efeito adicional, nos parâmetros citados, do metronidazol tópico, como já evidenciado por alguns estudos quando utilizado isoladamente ${ }^{8,9}$.

\section{MATERIAL E MÉTODOS}

Tratou-se de um estudo longitudinal prospectivo, randomizado, duplo-cego, realizado no serviço de Coloproctologia do Hospital de Base - Faculdade de Medicina de São José do Rio Preto, no período de abril de 2009 a agosto de 2010.

O estudo foi aprovado por Comitê de Ética em Pesquisa (CEP) local e a assinatura do Termo de Consentimento Livre e Esclarecido foi obtida de cada participante do estudo, após esclarecimento do mesmo.

Para o estudo, os pacientes foram divididos em dois grupos aleatórios, definidos por sorteio numerado no momento da cirurgia: grupo 1 (NIF), que utilizou apenas nifedipina tópica $0,3 \%$, e grupo 2 (NIF+METRO), que utilizou a associação nifedipina tópica $0,3 \%$ e metronidazol $10 \%$.

Os pacientes receberam alta hospitalar no mesmo dia do procedimento cirúrgico, em tempo inferior às 12 horas iniciais.

No pós-operatório, os participantes foram orientados a realizar medidas higieno-dietéticas; dipirona $1 \mathrm{~g}$, via oral, se dor leve, até $4 \mathrm{x} /$ dia; cetorolato $10 \mathrm{mg}$, sublingual, se dor forte ou refratariedade à dipirona, até $4 \mathrm{x} / \mathrm{dia}$; banhos de assento com água morna e permanganato de potássio, 20 minutos, três vezes ao dia seguido da aplicação tópica de nifedipina $0,3 \%+$ metronidazol $10 \%$ ou nifedipina $0,3 \%$ isolada, a partir de período anterior às 12 horas iniciais após cirurgia até 28 dias desta.

Os critérios de inclusão foram: persistência de sintomatologia (dor, prolapso, hematoquezia) após o tratamento clínico e/ou conservador, pacientes incapazes de tolerar o tratamento conservador e que não o desejam, independente do grau da hemorroida, além de portadores de hemorroidas sintomáticas não volumosas (graus 3/4) ou mistas.

Da mesma forma, foram considerados critérios de exclusão: cirurgias prévias anorretais, complicações agudas da doença hemorroidária, outra doença anorretal concomitante, gestantes, déficit neurológico, síndrome dolorosa crônica, comorbidades inca- 
pacitantes, alergia a um dos componentes do estudo, nifedipina e/ou metronidazol, bem como uso do antihipertensivo nifedipina via oral.

\section{Técnica cirúrgica}

Pacientes realizaram enema evacuador no préoperatório (dia anterior, à noite). Não foi realizado antibioticoprofilaxia na indução anestésica. A anestesia durante o ato cirúrgico foi a raquianestesia "em sela" e local com $20 \mathrm{~mL}$ de bupivacaína $0,5 \%$ com vasoconstritor, distribuídos nos quatro quadrantes. Os pacientes foram operados pelo mesmo cirurgião.

Os pacientes foram posicionados em decúbito ventral com coxim subpúbico e submetidos à hemorroidectomia eletiva pela técnica híbrida com uso de fio poliglecaprone 5.0 (Caprofyl ${ }^{\circledR}$ ou Monocryl).

\section{Coleta dos dados}

Foram colhidos dados demográficos (sexo, idade), dados referentes à dor, conforme nota baseada numa escala visual de 0 a 10 (Figura 1) e dose necessária de medicação, evacuação, presença de plicoma inflamatório e complicações cirúrgicas nas primeiras 12 horas, $1^{\circ}, 4^{\circ}, 14^{\circ}$ e $28^{\circ}$ dia pós-operatório, nos retornos ambulatoriais nas mesmas datas.

\section{Análise estatística}

Foram utilizadas estatísticas descritivas com abordagem de testes estatísticos não-paramétricos como teste de Mann-Whitney e teste de KruskalWallis, além do teste de comparação múltipla de Dunn, todos com nível de significância de 0,05 . Ademais, foi utilizado o teste $\chi^{2}$, com o objetivo de se verificar associação entre as variáveis coletadas.

\section{RESULTADOS}

Um total de 42 pacientes foi submetido à cirurgia de hemorroidectomia, divididos em dois grupos: 21 pa-

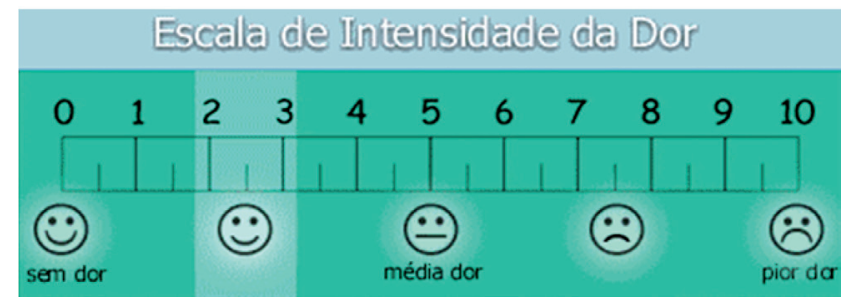

Figura 1. Escala Visual de Dor. cientes tratados com nifedipina $0,3 \%$ (GRUPO 1 - NIF) e 21 pacientes tratados com nifedipina $0,3 \%$ e metronidazol 10\% (GRUPO 2 - NIF+METRO). Houve abandono do tratamento e/ou não retorno às consultas de avaliação em três casos no grupo NIF+METRO e quatro casos no grupo NIF; porém, esses pacientes não foram excluídos e seus dados coletados utilizados nas análises (Figura 2).

O grupo NIF consistiu-se de 13 pacientes $(61,90 \%)$ do sexo feminino e $8(38,10 \%)$ do sexo masculino. Com relação à idade, verificou-se que a idade média dos pacientes foi de 50,10 anos com desvio padrão de 12,20 anos e a mediana de 51 anos. O grupo NIF+METRO apresentou 10 pacientes $(47,62 \%)$ do sexo feminino e $11(52,38 \%)$ do sexo masculino. A idade média dos pacientes foi de 45,33 anos com desvio padrão de 12,10 anos e a mediana de 45 anos (Tabela 1).

Além disso, realizou-se uma análise descritiva percentual para cada grupo amostral, em relação ao número de mamilos hemorroidários operados nos pacientes envolvidos no estudo (Tabela 2), e a relação entre

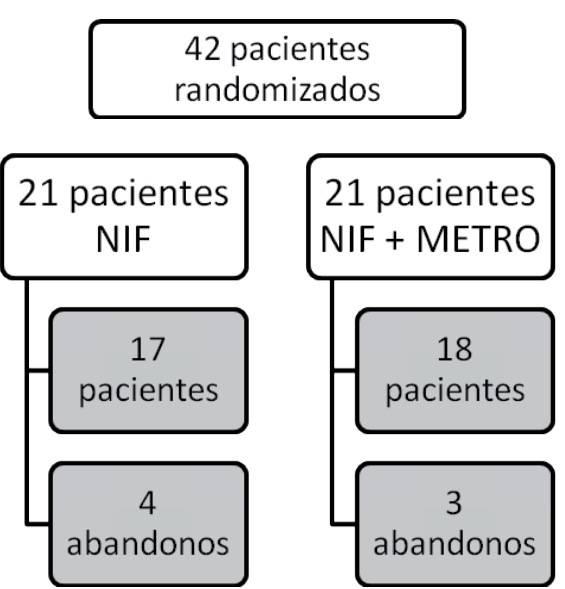

Figura 2. Fluxograma dos pacientes randomizados no estudo. NIF: grupo 1, que utilizou apenas nifedipina tópica 0,3\%; NIF+METRO: grupo 2, que utilizou a associação nifedipina tópica $0,3 \%$ e metronidazol $10 \%$.

Tabela 1. Dados demográficos dos grupos.

\begin{tabular}{lcc}
\hline Estatísticas & NIF & NIF+METRO \\
\hline Média & 50,1 & 45,33 \\
Desvio padrão & 12,20 & 12,10 \\
Mediana & 51 & 45 \\
Variação de idade & $18-70$ & $24-67$ \\
Razão M/F & $8 / 13$ & $11 / 10$ \\
\hline
\end{tabular}

NIF: grupo 1, que utilizou apenas nifedipina tópica 0,3\%; NIF+METRO: grupo 2, que utilizou a associação nifedipina tópica $0,3 \%$ e metronidazol $10 \%$. 
o número de mamilos ressecados e a dor relatada após o procedimento cirúrgico, estratificados por grupos amostrais (Tabela 3).

Não houve diferença estatística entre os grupos, em relação aos dados demográficos, distribuição percentual dos mamilos operados e da dor, em relação ao número de mamilos ressecados. Apesar de não ter sido possível pressupor diferenças entre os grupos amostrais em relação à dor, verificou-se que os escores foram maiores para os pacientes que apresentaram um mamilo retirado.

As demais análises estatísticas foram fundamentadas na verificação das variáveis coletadas acerca das condições dos pacientes avaliados para cada período pósoperatório citado. As variáveis abordadas no estudo são: dor pós-operatória, necessidade de uso de analgésicos e número de comprimidos ingeridos, evacuação, presença de plicoma inflamatório e complicações.

\section{Dor pós-operatória}

Na avaliação da dor, foi possível verificar que o grupo NIF, em média, apresentou maior dor quando comparado ao grupo NIF+METRO, principalmente nas 12 horas iniciais, $1^{\circ}$ e $4^{\circ}$ dia pós-operatório. A dor no $14^{\circ} \mathrm{e}$ $28^{\circ}$ dia foram praticamente inexistentes. Além disso, a mediana do grupo. NIF foi superior à mediana do grupo NIF+METRO, reiterando a constatação desse fato. Apesar da diferença entre as medianas ser considerável, o valor $\mathrm{p}$ encontrado para o teste de Mann-Whitney foi superior ao nível de significância adotado, pressupondo a ausência de diferenças estatisticamente significativas entre os grupos quanto à dor (Tabela 4).

Ao analisar-se a dor relativa aos períodos pós-operatórios, verificou-se a existência de diferenças estatisticamente significativas nos escores de dor, quando os períodos pós-operatórios são comparados. A Tabela 5 mostra as estatísticas descritivas dos escores da dor, de acordo com cada período pós-operatório avaliado para os dois grupos NIF e NIF+METRO.
Fazendo-se a análise do grupo NIF, isoladamente, foi possível verificar-se, pelos resultados do teste de comparação múltipla de Dunn, que os escores de dor dos períodos de 12 horas, $1^{\circ}$ pós-operatório (PO) e $4^{\circ} \mathrm{PO}$ diferem significativamente dos períodos $14^{\circ}$ $\mathrm{PO}$ e $28^{\circ} \mathrm{PO}$, sendo esses últimos os que apresentaram menores escores. Já, para o grupo NIF+METRO, a maior discrepância está entre os escores referentes aos períodos de 12 horas e $28^{\circ} \mathrm{PO}$, sendo os escores de dor superiores no primeiro período.

A Figura 3 mostra a evolução dos valores de mediana da dor ao longo dos períodos avaliados para ambos os grupos amostrais.

Verificou-se que em todos os períodos, exceto no $14^{\circ} \mathrm{PO}$ e $28^{\circ} \mathrm{PO}$, os escores de dor do grupo NIF são superiores ao grupo NIF+METRO, ou seja, o tratamento de uso tópico NIF+METRO fornece indícios de ser mais eficaz contra a dor.

\section{Uso de analgésicos e número de comprimidos ingeridos}

Ao analisar os grupos de forma isolada, verificou-se, na maioria das avaliações, que o grupo NIF apresentou maior consumo e dose medicamentosa que o grupo NIF+METRO. Porém, essa discrepância entre os grupos não foi suficiente para resultar em associação do consumo e dose de analgésicos em relação aos grupos amostrais, visto que o valor $\mathrm{p}$ encontrado foi superior a 0,05 .

Logo, nota-se ausência de associação entre o uso de analgésico comum e potente e o tratamento tópico diferenciado. Além disso, não há pressuposições acerca da existência de associação entre a dose de analgésicos e os grupos amostrais.

\section{Evacuação e plicoma inflamatório}

O valor p encontrado para o teste de associação mostra que, a precocidade da evacuação e o aparecimento de

Tabela 2. Distribuição percentual do número de mamilos em relação aos grupos avaliados no estudo.

\begin{tabular}{lccc}
\hline Número de mamilos & NIF & NIF+METRO & Total \\
\hline 1 & $7(33,33 \%)$ & $11(52,38 \%)$ & $18(42,86 \%)$ \\
2 & $13(61,90 \%)$ & $9(42,86 \%)$ & $21(52,38 \%)$ \\
3 & $1(4,76 \%)$ & $1(4,76 \%)$ & $2(4,76 \%)$ \\
Total & $21(50,00 \%)$ & $21(50,00 \%)$ & $42(100 \%)$ \\
\hline
\end{tabular}

NIF: grupo 1, que utilizou apenas nifedipina tópica $0,3 \%$;

NIF+METRO: grupo 2, que utilizou a associação nifedipina tópica 0,3\% e metronidazol 10\%. 
Tabela 3. Estatísticas descritivas da dor em relação ao número de mamilos. Valor p referente ao teste de Mann-Whitney.

\begin{tabular}{|c|c|c|c|c|c|c|}
\hline $\begin{array}{l}\mathrm{N}^{0} \text { de } \\
\text { mamilos }\end{array}$ & Período & Grupo & $\mathbf{N}$ & $\bar{x} \pm s$ & $\mathbf{M d}^{*}$ & Valor $\mathbf{p}$ \\
\hline \multirow[t]{10}{*}{ 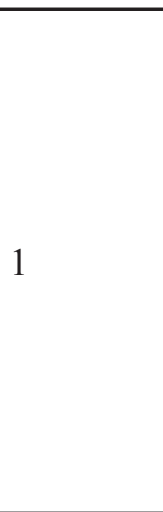 } & \multirow{2}{*}{$12 \mathrm{~h}$} & NIF & 7 & $6,86 \pm 4,10$ & 9,00 & \multirow{2}{*}{0,123} \\
\hline & & NIF+METRO & 11 & $4,63 \pm 3,00$ & 4,00 & \\
\hline & \multirow{2}{*}{$1 \mathrm{PO}$} & NIF & 7 & $5,57 \pm 2,57$ & 5,00 & \multirow{2}{*}{0,057} \\
\hline & & NIF+METRO & 11 & $3,18 \pm 2,63$ & 4,00 & \\
\hline & \multirow{2}{*}{$4 \mathrm{PO}$} & NIF & 6 & $4,50 \pm 3,83$ & 5,00 & \multirow{2}{*}{0,398} \\
\hline & & NIF+METRO & 9 & $3,11 \pm 2,84$ & 4,00 & \\
\hline & \multirow{2}{*}{$14 \mathrm{PO}$} & NIF & 5 & $1,70 \pm 3,80$ & 0,00 & \multirow{2}{*}{0,788} \\
\hline & & NIF+METRO & 8 & $2,00 \pm 3,07$ & 0,00 & \\
\hline & \multirow{2}{*}{$28 \mathrm{PO}^{*}$} & NIF & 5 & $1,00 \pm 1,41$ & 0,00 & \multirow[b]{2}{*}{-} \\
\hline & & NIF+METRO & 8 & $0,00 \pm 0,00$ & 0,00 & \\
\hline \multirow{10}{*}{2} & \multirow{2}{*}{$12 \mathrm{~h}$} & NIF & 13 & $5,92 \pm 3,47$ & 5,00 & \multirow{2}{*}{0,664} \\
\hline & & NIF+METRO & 9 & $5,11 \pm 3,37$ & 5,00 & \\
\hline & \multirow{2}{*}{$1 \mathrm{PO}$} & NIF & 13 & $4,69 \pm 2,25$ & 5,00 & \multirow{2}{*}{0,789} \\
\hline & & NIF+METRO & 9 & $4,33 \pm 3,28$ & 5,00 & \\
\hline & \multirow{2}{*}{$4 \mathrm{PO}$} & NIF & 13 & $3,46 \pm 2,69$ & 3,00 & \multirow{2}{*}{0,664} \\
\hline & & NIF+METRO & 9 & $2,88 \pm 2,37$ & 3,00 & \\
\hline & \multirow{2}{*}{$14 \mathrm{PO}$} & NIF & 12 & $0,75 \pm 1,54$ & 0,00 & \multirow{2}{*}{1,000} \\
\hline & & NIF+METRO & 9 & $0,88 \pm 1,83$ & 0,00 & \\
\hline & \multirow{2}{*}{$28 \mathrm{PO}^{*}$} & NIF & 12 & $0,00 \pm 0,00$ & 0,00 & \multirow{2}{*}{-} \\
\hline & & NIF+METRO & 9 & $0,00 \pm 0,00$ & 0,00 & \\
\hline
\end{tabular}

NIF: grupo 1, que utilizou apenas nifedipina tópica 0,3\%;

NIF+METRO: grupo 2, que utilizou a associação nifedipina tópica 0,3\% e metronidazol 10\%. PO: pós-operatório; Md: média.

* Não foi possivel obter um valor p referente ao $28^{\circ} \mathrm{PO}$, devido aos pacientes do grupo NIF + METRO terem avaliado a dor com escores nulos.

Tabela 4. Estatísticas descritivas da dor de acordo com os grupos amostrais avaliados. Valor p referente ao teste de Mann-Whitney.

\begin{tabular}{|c|c|c|c|c|c|c|c|c|}
\hline Período & Grupo & $\mathbf{N}$ & \pm & Md & Mín & Máx & IC (95\%) & Valor $p$ \\
\hline \multirow[t]{2}{*}{$12 \mathrm{~h}$} & NIF & 21 & $6,42 \pm 3,62$ & 8,00 & 0,00 & 10,00 & $(4,000 ; 9,326)$ & \multirow{2}{*}{0,074} \\
\hline & NIF+METRO & 21 & $4,66 \pm 3,13$ & 5,00 & 0,00 & 10,00 & $(2,673 ; 7,326)$ & \\
\hline \multirow[t]{2}{*}{$1 \mathrm{PO}$} & NIF & 21 & $5,14 \pm 2,37$ & 5,00 & 1,00 & 9,00 & $(3,673 ; 7,000)$ & \multirow{2}{*}{0,078} \\
\hline & NIF+METRO & 21 & $3,71 \pm 2,84$ & 4,00 & 0,00 & 10,00 & $(1,673 ; 5,000)$ & \\
\hline \multirow[t]{2}{*}{$4 \mathrm{PO}$} & NIF & 20 & $4,00 \pm 3,09$ & 4,50 & 0,00 & 9,00 & $(1,235 ; 6,764)$ & \multirow{2}{*}{0,216} \\
\hline & NIF+METRO & 19 & $2,84 \pm 2,56$ & 3,00 & 0,00 & 8,00 & $(1,605 ; 4,078)$ & \\
\hline \multirow[t]{2}{*}{$14 \mathrm{PO}$} & NIF & 17 & $1,03 \pm 2,33$ & 0,00 & 0,00 & 8,50 & $(0,000 ; 0,000)$ & \multirow{2}{*}{0,791} \\
\hline & NIF+METRO & 18 & $1,33 \pm 2,42$ & 0,00 & 0,00 & 8,00 & $(0,000 ; 1,446)$ & \\
\hline \multirow[t]{2}{*}{$28 \mathrm{PO}^{*}$} & NIF & 17 & $0,29 \pm 0,84$ & 0,00 & 0,00 & 3,00 & - & \multirow{2}{*}{ - } \\
\hline & NIF+METRO & 18 & $0,00 \pm 0,00$ & 0,00 & 0,00 & 0,00 & - & \\
\hline
\end{tabular}

NIF: grupo 1, que utilizou apenas nifedipina tópica $0,3 \%$;

NIF+METRO: grupo 2, que utilizou a associação nifedipina tópica 0,3\% e metronidazol 10\%. PO: pós-operatório; Md: média.

*Todos os pacientes do grupo NIF+METRO apresentavam dor ausente no $28^{\circ} \mathrm{PO}$. 
Marcelo Pandolfi Basso e Cols.

Tabela 5. Estatísticas descritivas da dor em relação aos periodos pós-operatório. Valor p referente ao teste de Kruskal-Wallis.

\begin{tabular}{lcccccrc}
\hline Grupo & Período & $\mathbf{N}$ & $\bar{x} \pm s$ & Md $^{*}$ & Mín & Máx & Valor \\
\hline \multirow{4}{*}{ NIF } & $12 \mathrm{~h}$ & 21 & $6,43 \pm 3,62$ & $8,00^{\mathrm{A}}$ & 0,00 & 10,00 & \\
& $1 \mathrm{PO}$ & 21 & $5,14 \pm 2,37$ & $5,00^{\mathrm{A}}$ & 1,00 & 9,00 & \\
& $4 \mathrm{PO}$ & 20 & $4,00 \pm 3,09$ & $4,50^{\mathrm{A}}$ & 0,00 & 9,00 & $<0,001$ \\
& $14 \mathrm{PO}$ & 17 & $1,03 \pm 2,33$ & $0,00^{\mathrm{B}}$ & 0,00 & 8,50 & \\
& $28 \mathrm{PO}$ & 17 & $0,29 \pm 0,84$ & $0,00^{\mathrm{B}}$ & 0,00 & 3,00 & \\
\multirow{3}{*}{ NIF+METRO } & $12 \mathrm{~h}$ & 21 & $4,66 \pm 3,13$ & $5,00^{\mathrm{A}}$ & 0,00 & 10,00 & \\
& $1 \mathrm{PO}$ & 21 & $3,71 \pm 2,85$ & $4,00^{\mathrm{AB}}$ & 0,00 & 10,00 & \\
& $4 \mathrm{PO}$ & 19 & $2,84 \pm 2,56$ & $3,00^{\mathrm{AB}}$ & 0,00 & 8,00 & $<0,001$ \\
& $14 \mathrm{PO}$ & 18 & $1,33 \pm 2,42$ & $0,00^{\mathrm{BC}}$ & 0,00 & 8,00 & \\
\hline
\end{tabular}

NIF: grupo 1, que utilizou apenas nifedipina tópica $0,3 \%$;

NIF+METRO: grupo 2, que utilizou a associação nifedipina tópica 0,3\% e metronidazol 10\%. PO: pós-operatório; Md: média.

*Letras diferentes na mesma coluna diferem significativamente a 0,05 pelo teste de comparação múltipla de Dunn.

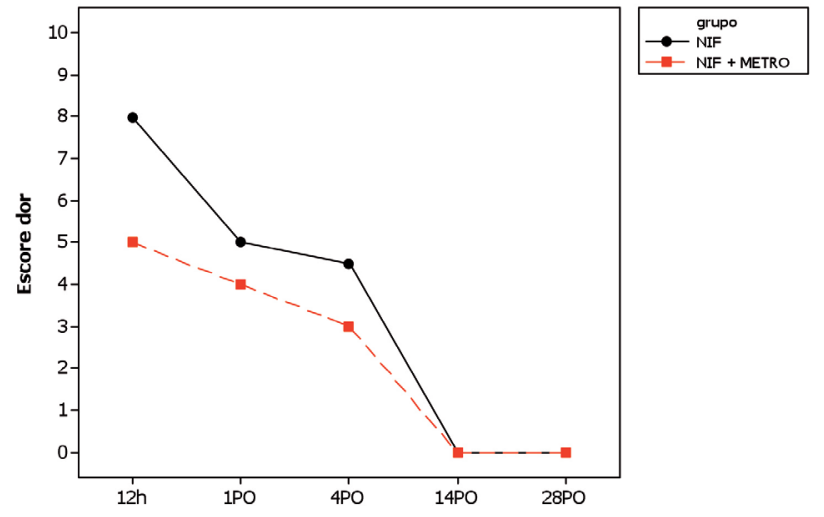

Figura 3. Escores de dor ao longo dos períodos pós-operatório. NIF: grupo 1, que utilizou apenas nifedipina tópica $0,3 \%$;

NIF+METRO: grupo 2, que utilizou a associação nifedipina tópica $0,3 \%$ e metronidazol $10 \%$.

plicoma inflamatório não estão relacionados ao tratamento diferenciado dado aos dois grupos amostrais referente aos dias de análise, visto que os valores $\mathrm{p}$ encontrados foram superiores a 0,05 .

Ocorreram 14 casos com plicoma inflamatório no grupo NIF, com 1 regressão no período avaliado, enquanto no grupo NIF + METRO ocorreram 9 casos, sem regressões.

\section{Complicações}

Um total de 12 complicações em 9 pacientes estavam presentes no grupo NIF: 1 retenção urinária, 1 hematoma, 4 trombos hemorroidários, 2 equimoses,
1 edema da ferida cirúrgica, 2 deiscências de sutura e 1 fissura anal. No grupo NIF+METRO observaramse 7 complicações em 5 pacientes: 1 hematoma, 2 equimoses, 2 deiscências, 1 trombo e 1 fissura.

Não é possível verificar a existência de associação significativa entre a existência de complicações e os grupos amostrais avaliados, devido ao valor $\mathrm{p}$ encontrado ter resultado igual a 0,190 . Apesar disso, o tratamento tópico ministrado ao grupo NIF+METRO foi mais eficaz, visto que apresentou menor número de pacientes com complicações.

\section{DISCUSSÃO}

A dor no pós-operatório de hemorroidectomia implica em desconforto e sofrimento. A gênese da dor não é bem definida. O papel do espasmo esfincteriano e colonização bacteriana como contribuinte desta não foi totalmente elucidado ${ }^{9,10}$.

Diversos procedimentos e técnicas cirúrgicas, como a hemorroidopexia com uso de grampeador circular tem demonstrado redução desta e um retorno mais rápido para atividades cotidianas ${ }^{3,6}$. No entanto, seu alto custo limita seu uso rotineiro. $\mathrm{O}$ uso de medicação oral e tópica com objetivo de alívio álgico torna-se mais factível e acessível aos médicos e pacientes.

Vários estudos avaliaram o uso de metronidazol e a dor no pós-operatório de hemorroidectomia. Seu uso oral apresenta resultados conflitantes, com benefício 
duvidoso ${ }^{6,7,9}$. Em 1998, Carapeti et al. ${ }^{7}$ demonstraram, em estudo prospectivo e randomizado, que o metronidazol oral significativamente diminuiu a dor pós operatória nos dias 5, 6 e 7 após hemorroidectomia aberta por diatermia, além de determinar melhor satisfação do paciente e retorno precoce ao trabalho. Resultado de estudo subsequente, Balfour et al. ${ }^{6}$ não mostraram diferenças entre os grupos que usaram metronidazol oral após hemorroidectomia técnica fechada.

Porém, quando se avalia o metronidazol tópico a $10 \%$, diversos estudos mostram melhora da dor, do desconforto evacuatório e dos índices de cicatrização e edema ${ }^{8,9}$. O uso tópico apresenta este melhor efeito devido aumento da concentração tecidual local, menores efeitos colaterais sistêmicos e melhor biodisponibilidade 9 .

Ademais, o uso de nitratos e bloqueadores de canal de cálcio, dentre estes a nifedipina tópica, também relaciona-se com menor dor no pós-operatório, menor necessidade de uso de analgésicos e melhor cicatrização. A limitação ao uso da nitroglicerina tópica é a cefaleia decorrente do seu uso ${ }^{1,4,5,10,11}$.

No estudo em questão, observou-se uma diminuição da dor pós-operatória, de acordo com a escala visual utilizada, além de menor uso da medicação analgésica comum e potente e menor dose medicamentosa necessária para alívio desta, nos períodos avaliados, quando comparamos o uso combinado de nifedipina $0,3 \%$ e metronidazol $10 \%$ tópico em relação a nifedipina $0,3 \%$ tópica isolada. Apesar disso, na avaliação estatística não obtivemos resultados significantes nestes parâmetros.

Quando os períodos são comparados entre si, segundo teste de comparação de Dunn, o grupo NIF+METRO demonstra diferença de dor apenas nas 12 horas iniciais e $28^{\circ} \mathrm{PO}$, sem diferenças entre o $1^{\circ}, 4^{\circ}$ e $14^{\circ} \mathrm{PO}$. De forma diferente, no grupo NIF, as 12 horas iniciais, o $1^{\circ}$ e $4^{\circ} \mathrm{PO}$ não diferem entre si, diferindo apenas do $14^{\circ}$ e $28^{\circ} \mathrm{PO}$, períodos estes com medianas bem inferiores às demais nos dois grupos. Isso reitera o fato de que, no caso de medicações associadas a dor nos períodos iniciais, como o $1^{\circ}$ e $4^{\circ} \mathrm{PO}$, foram semelhantes a período com mediana baixa, como o $14^{\circ} \mathrm{PO}$. Estas diferenças foram estatisticamente significantes.

Por fim, ambos os grupos não diferiram estatisticamente com relação à precocidade da evacuação, desenvolvimento de plicomas inflamatórios e complicações, apesar de menos frequentes no grupo de medicações combinadas.

Provavelmente, o principal motivo para os resultados acima citados foi o número insuficiente de pacientes participantes.

\section{CONCLUSÃO}

A doença hemorroidária apresenta grande prevalência e seu tratamento cirúrgico é causa de dor de difícil controle e sem um tratamento totalmente eficaz e acessível. A avaliação da dor pós-operatória, necessidade de uso de analgésicos, evacuação, presença de plicoma inflamatório e complicações de pacientes submetidos à hemorroidectomia pela técnica híbrida, que usaram nifedipina tópica $0,3 \%$ associada ou não ao metronidazol $10 \%$, no periodo pós-cirúrgico de 28 dias, não demonstrou diferença estatisticamente significante. Apesar disso, ambos os tratamentos são justificáveis de serem utilizados, pelos benefícios demonstrados na literatura médica da nifedipina e metronidazol tópicos no pós-operatório de hemorroidectomia.

\footnotetext{
ABSTRACT: Introduction: Hemorrhoidectomy is a frequent operation considered painful for most patients. Topical metronidazole and nifedipine have been demonstrated to decrease postoperative pain when they are applied alone. The aim of this study was to evaluate the effect of topical metronidazole and nifedipine together in reducing postoperative pain, in the evacuation, the development of inflammatory skin tags and complications. Material and methods: A double-blind, prospective, randomized study including 42 patients was performed. The group 1 used $10 \%$ metronidazole plus $0.3 \%$ nifedipine and the group 2 used $0.3 \%$ nifedipine only. Pain was assessed using a visual analog score (VAS) on postoperative hour 12 and at days 1, 4, 14 and 28. Results: Patients who used the combination of topical medications had less pain, less analgesic requirement and a lower dose ingested of analgesics, higher early defecation, lower development of inflammatory skin tags and complications than those who used topical nifedipine alone. Nevertheless, statistic differences were not significant. Conclusion: The addition of metronidazole to nifedipine in topical form did not improve the parameters evaluated in this study. However, both treatments are justified for use in postoperative hemorrhoidectomy.
}

Keywords: pain, postoperative; metronidazole; hemorrhoids; hemorrhoidectomy. 


\section{REFERÊNCIAS}

1. Perrotti P, Antropoli C, Noschese G, Domenico M, Antropoli M, Balzano A, et al. Topical Nifedipine ${ }^{\circledR}$ for conservative treatment of acute haemorrhoidal thrombosis. Colorectal Dis 2000;2(1):18-21.

2. Nivatvongs, S. Hemorrhoids. In: Gordon PH, Nivatvongs $\mathrm{S}$, editors. Principles and practice of surgery for the colon, rectum, and anus. 3rd ed. New York: Informa Healthcare; 2007. p. 144-64.

3. Longo A. Treatment of hemorrhoidal disease by reduction of mucosa and hemorrhoidal prolapse with a circular suturing device: a new procedure. In: Proceeding of the 6th world congress of endoscopic surgery. Rome. Surg Endosc 1998;12(5):777-84.

4. Wasvary HJ, Hain J, Mosed-Vogel M, Bendick P, Barkel DC, Klein SN. Randomized, prospective, double-blind, placebocontrolled trial of effect of nitroglycerin ointment on pain after hemorrhoidectomy. Dis Colon Rectum 2001;44(8):1069-73.

5. Elton $\mathrm{C}$, Sen $\mathrm{P}$, Montgomery AC. Initial study to assess the effects of topical glyceryl trinitrate for pain after haemorrhoidectomy. Int J Surg Investig 2001;2(5):353-7.

6. Balfour L, Stojkovic SG, Botterill ID, Burke DA, Finan PJ, Sagar PM. A randomized, double-blind trial of the effect of metronidazole on pain after closed hemorrhoidectomy. Dis
Colon Rectum 2002;45(9):1186-90.

7. Carapeti EA, Kamm MA, McDonald PJ, Phillips RK. Double-blind randomized controlled trial of effect of metronidazole on pain after day-case haemorrhoidectomy. Lancet 1998;351(9097):169-72.

8. Ala S, Saeedi M, Eshghi F, Mirzabeygi P. Topical metronidazole can reduce pain after surgery and pain on defecation in postoperative hemorrhoidectomy. Dis Colon Rectum 2008;51(2):235-8.

9. Nicholson TJ, Armstrong D. Topical metronidazole (10 percent) decreases posthemorrhoidectomy pain and improves healing. Dis Colon Rectum 2004;47(5):711-6.

10. Cesar MAP, Klug WA, Bassi DG, Paula PR, Cesar RP, Ortiz $\mathrm{JA}$, et al. Efeito da nifedipina gel $0,2 \%$ nas pressões de canal anal e na dor pós-operatória: estudo após hemorroidectomia pela técnica aberta. Rev bras Coloproct 2007;27(4):364-73.

11. Soltany S, Toussy JA, Fard MF. The effect of $0.2 \%$ glyceryl trinitrate ointment on pain and wound healing after hemorrhoidectomy. Surgery J 2009;4(1):8-12.

Endereço para correspondência:

João Gomes Netinho

Rua San Francisco, 481 Condomínio Débora Cristina - CEP: 15093-030 - São José do Rio Preto (SP), Brasil

E-mail: jgnetinho@riopreto.com.br 